Haben Sie Fragen zur Abrechnung oder zur wirtschaftlichen Praxisführung? Als Leser der MMW können Sie sich an unseren Experten wenden: Helmut Walbert, Facharzt für Allgemeinmedizin und Betriebswirt aus Würzburg.

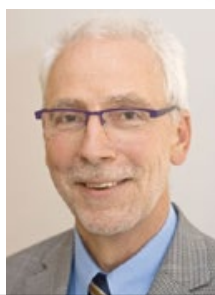

Helmut Walbert

Allgemeinarzt,

Medizinjournalist und

Betriebswirt Medizin

\title{
Apotheken-Empfehlung ist meist illegal!
}

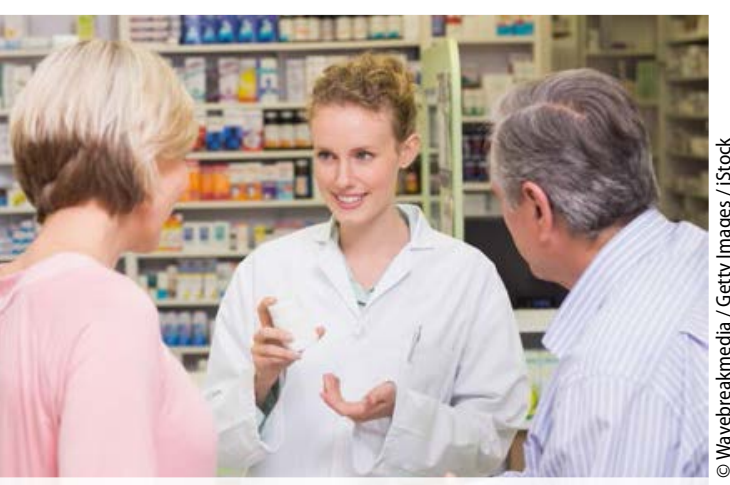

Nur spezialisierte Apotheken darf man empfehlen.
! MMW-Experte Walbert: Nicht erst das neue Gesetz macht Absprachen zwischen Ärzten und Apothekern sowie ähnlichen Leistungserbringern in den meisten Fällen illegal. Ihre vertragliche Verknüpfung macht die Situation noch kritischer.

Der Bundesgerichtshof (BGH) hat in verschiedenen Urteilen zur Empfehlung bestimmter Leistungserbringer klar Stellung genommen (z.B. Az.: I ZR 111/08, Az.: I ZR 275/99). Ärzte müssen penibel darauf achten, dass sie Patienten gegenüber niemals einer Apotheke den Vorzug geben. Dies verbietet nicht zuletzt die Berufsordnung. Natürlich kann das schwierig sein, wenn der Apotheker gleichzeitig der Vermieter ist.

Nur bei einem „hinreichenden Grund“ kann von der Regel abgewichen werden, etwa wenn der Patient ausdrücklich danach fragt und der Arzt seiner Fürsorgepflicht nachkommen will. Das gilt insbesondere dann, wenn sich eine Apotheke etwa mit ihrem Sortiment und der Fortbildung der Mitarbeiter auf bestimmte Krankheitsbilder (z.B. Diabetes) oder Heilmittelmethoden spezialisiert hat. Diesen „Mehrwert“ muss der Patient dem Arzt aber rückmelden, da der BGH fordert, dass eine Empfehlung auf „nachprüfbaren und aussagekräftigen Erfahrungen des Arztes“ beruht.

Sie sollten außerdem den Mietvertrag kritisch überprüfen (lasssen). Er könnte Punkte enthalten, die für einen formulierten wirtschaftlichen Vorteil für einen oder beide Vertragspartner sprechen also einen schriftlichen Beweis für strafbares Verhalten.

\section{Beim Medikationsplan hilft die PRISCUS-Liste}

$?$ Dr. I. P., Allgemeinärztin, Bayern: Für den neuen Medikationsplan gehen wir die bisherige Medikation unserer älteren Patienten kritisch durch. Gibt es eine Hilfestellung zur Beurteilung von Nebenwirkungen und Interaktionen?

Antwort: Kritiker behaupten, dass ca. $40 \%$ der Altenheimbewohner Medikamente mit erheblichen potenziellen Nebenwirkungen erhalten. Der
Medikationsplan wird hoffentlich vielen Verordnern Anlass sein, das einmal kritisch zu überprüfen!

Hierbei kann eine übersichtliche, zwei Seiten lange Handreichung sehr hilfreich sein: die PRISCUS-Liste. Sie enthält eine Sammlung von in Deutschland erhältlichen Arzneimitteln, die für ältere Patienten potenziell ungeeignet sind und die daher bei Verordnung kritisch betrachtet werden sollten. Die Liste wurde 2010 von einem Forscherteam um die Wuppertaler Pharmakologin Petra A. Thürmann veröffentlicht. Ausgangspunkt war die Liste des amerikanischen Geriaters Mark H. Beers, der bereits 1991 Medikamente benannte, bei denen es insbesondere bei älteren Menschen vermehrt zu unerwünschten $\mathrm{Ne}$ benwirkungen kommen kann oder für die Alternativpräparate mit weniger $\mathrm{Ne}$ benwirkungen verfügbar sind.

-www.aok-gesundheitspart-ner.de/imperia/md/gpp/bund/ arztundpraxis/prodialog/2012/priscusliste_gpp.pdf 